\title{
The hairless (hr) gene is involved in the congenital hypotrichosis of Valle del Belice sheep
}

\author{
Raffaella FinOCCHIARO ${ }^{a *}$, Baldassare Portolano ${ }^{a}$, \\ Giuseppe Damiani ${ }^{\mathrm{b}}$, Anna $\mathrm{CAROLI}^{\mathrm{c}}$, Elena Budelli ${ }^{\mathrm{d}}$, \\ Patrizia Bolla $^{\mathrm{e}}$, Giulio PAgnaCCO ${ }^{\mathrm{e}}$ \\ ${ }^{a}$ Dipartimento S. En. Fi. Mi. Zo., Sezione Produzione Animale, Palermo, Italy \\ ${ }^{b}$ IGM-CNR, Pavia, Italy \\ c Dipartimento di Sanità e Benessere Animale, Valenzano (Bari), Italy \\ ${ }^{d}$ CERSA, Segrate (Milano), Italy \\ e Dipartimento di Veterinaria per la Sicurezza Alimentare, Milano, Italy
}

(Accepted 26 February 2003)

\begin{abstract}
Congenital hypotrichosis in mammalian species consists of partial or complete absence of hair at birth. The hairless gene is often responsible for this disorder in men, mice and rats. Recent experimental data on Valle del Belice sheep reared in Sicily for milk production, support the genetic control of the ovine hypotrichosis as a Mendelian recessive trait. The ovine hairless gene was chosen as the candidate gene involved in this disorder. Blood samples were collected from Valle del Belice sheep with the normal and hypotrichotic phenotypes. Almost the entire hairless gene was successfully amplified using the long PCR technique. Unrelated sheep with differing phenotypes were randomly chosen for sequencing the amplified products. Different mutations related to the hypotrichotic phenotype were found in exon 3. In fact, sequencing revealed an $\mathrm{A} / \mathrm{T}$ transversion at position 739 , a G/A transition at position 823 , and a $\mathrm{C} / \mathrm{T}$ transition at position 1312. From these nucleotide exchanges, three substitutions of the processed mature protein were deduced at the amino acid positions 247 ( $\mathrm{Thr} / \mathrm{Ser}$ ), 275 (Ala/ Thr), and 438 (Gln/Stop). A PCR-SSCP based test was developed in order to detect the last mutation, which is responsible for the hypotrichotic phenotype.
\end{abstract}

hairless gene / congenital hypotrichosis / ovine / Valle del Belice sheep

\section{INTRODUCTION}

Congenital hypotrichosis in mammalian species consists of a partial or complete absence of the hair coat at birth. The hairless ( $h r)$ gene is often responsible for this disorder. The gene is highly conserved between men,

\footnotetext{
* Correspondence and reprints
}

E-mail: rfino@unipa.it 
mice and rats $[1,2,6]$. The protein codified by this gene is a transcriptional corepressor for thyroid hormone receptors [13]. This gene is highly expressed in the brain and skin [1,4]. In its absence, hair follicles disintegrate and new hair is not induced [12]. Cachon-Gonzalez et al. [4] described the gene expression in mice, and amplified the entire cDNA of the gene, which consists of 19 exons with a coding sequence of 3750 nucleotides.

Experimental data suggest that the $h r$ gene product may play a crucial role in maintaining the delicate balance between cell proliferation, differentiation and apoptosis in the hair follicle, as well as in the interfollicular epidermis [2].

The literature reports a few cases of congenital hypotrichosis in the ovine breeds Australian Polled Dorset [7, 10], Karakul [11], and Valle del Belice [9]. So far no molecular study has been carried out in the ovine species. Experimental data on Valle del Belice, an ovine dairy breed reared in Sicily, support the genetic control of the anomaly as a Mendelian recessive trait, and demonstrate no concomitant chromosomal rearrangements [9]. The $h r$ gene was investigated as a candidate for genetic determinism of congenital hypotrichosis observed in the Valle del Belice breed.

\section{MATERIALS AND METHODS}

\subsection{Sampling and DNA extraction}

Peripheral blood samples were collected from Valle del Belice sheep with the normal $(\mathrm{n}=39)$ and hypotrichotic $(\mathrm{n}=16)$ phenotypes. Sheep were randomly chosen, avoiding close genetic relationship, except for two half-sibs with the normal phenotype. Genomic DNA was extracted from the blood by a DNA purification kit (GFX Genomic blood purification kit, Amersham Pharmacia Biotech).

\subsection{Primer design}

On the basis of the alignment of rat (accession number gi-13242257), mouse (accession number gi-11230771) and human (accession number gi-11036651) $h r$ mRNA sequences performed by the ClustalW program (www.ebi.ac.uk/clustalw), several conserved regions were identified and used to design primers for the amplification of almost the entire gene (Tab. I). Subsequently the obtained ovine sequences were used to design other primers for the sequencing reactions (Tab. I).

\subsection{PCR conditions}

The long PCR (TaKaRa LA Taq, TaKaRa Biomedicals) and PCR technique (RedTaq DNA Polymerase, Sigma) were used for the amplification which was performed by a PTC-200 Peltier Thermal Cycler (MJ Research). 
Table I. Primers for amplifying and sequencing different hairless gene regions using the long PCR ( $)$ or the normal PCR starting from genomic DNA $(*)$ or from the long PCR products.

\begin{tabular}{lll}
\hline \multicolumn{1}{c}{ Primer forward } & \multicolumn{1}{c}{ Primer reverse } & \multicolumn{1}{c}{$h r$ region } \\
\hline TTTAGGGCTTTACTACAAGGATC & TTGCAGGAAGGTTGTGGAGT & Exon 3-9 \\
CTGACCCAGTTTGTCTCCAGCCAG & GCCCAGAGCTGGGGCTCCAGTGGA & Exon 7-14 \\
AACTCAACCTGGCTTCCTACCTC & GCTCACCAGGCCCTGCACCT & Exon 14-18 \\
CCCATGAGGGCAGGAGAGTGAT & TAAGCTGAAGGCAGAGGGCACTTT & Exon $2^{*}$ \\
TTTAGGGCTTTTACTACAAGGATC & GCCCATTGCTCCCTGGACCTC & Exon 3 \\
CTGGAGCGAGCTCCCTTCTG & TTCCACAGTTGCCAATGGGTCC & Intron $2^{*}$ \\
GCCCAGCTGCCAGCCCGCAA & ACAGTGGCTGGGAGTAGTGGGC & Intron 3* \\
TGTGCCCAGGCAGCTGGAGAG & TCGCCAGAGGGTGTTGAAGAGTC & Exon 4-6 Intron 4-5 \\
ACAGAGGACAGCCCAGGCATTCCA & CAAACTTGACCCAGACCTGGTGCAT & Exon 6-8 Intron 6-7 \\
CTGACCCAGTTTGTCTCCAGCCAG & TTGCAGGAAGGTTGTGGAGT & Exon 7-9 Intron 7-8 \\
GTCAAACTCTGCTTGGGGCATGA & GCCCAGAGCTGGGGCTCCAGTGGA & Exon 10-14 Intron 10-13 \\
AACTCAACCTGGCTTCCTACCTC & ATCTGGAGAAAGCGGCGGATGC & Exon 14, 15* Intron 14 \\
GACACCTGGGGACCAAGAACCT & ACCAGCACGGCCTCTCCGGG & Exon 15-17 Intron 15, 16 \\
\hline
\end{tabular}


Long PCR was performed in $20 \mu \mathrm{L}$ containing $40-80$ ng genomic DNA, $1 \mu \mathrm{M}$ forward and reverse primers (Tab. I), 1 unit of TaKaRa LA Taq, $2.5 \mathrm{mM}$ $\mathrm{MgCl}_{2}, 2.5 \mu \mathrm{M}$ dNTP mixture and the LA PCR buffer. After an initial denaturation of $2 \mathrm{~min}$ at $94^{\circ} \mathrm{C}$, samples were subjected to 30 cycles of $20 \mathrm{~s}$ at $98^{\circ} \mathrm{C}, 30 \mathrm{~s}$ at $55^{\circ} \mathrm{C}$, and $10-20 \mathrm{~min}$ at $68^{\circ} \mathrm{C}$, followed by a final elongation of $10 \mathrm{~min}$ at $72^{\circ} \mathrm{C}$.

PCR was performed in $20 \mu \mathrm{L}$ containing $40-80 \mathrm{ng}$ of genomic DNA or PCR products diluted 1:100 as template, $0.4 \mu \mathrm{M}$ forward and reverse primers (Tab. I), 1 unit of RedTaq DNA polymerase, $2 \mu \mathrm{M}$ dNTP mixture and the REDTaq PCR buffer. After an initial denaturation of $2 \mathrm{~min}$ at $94^{\circ} \mathrm{C}$, samples were subjected to 35 cycles of $30 \mathrm{~s}$ at $94^{\circ} \mathrm{C}, 30 \mathrm{~s}$ at $55^{\circ} \mathrm{C}-65^{\circ} \mathrm{C}$, and $2-10 \mathrm{~min}$ at $72^{\circ} \mathrm{C}$, followed by a final elongation step of $5 \mathrm{~min}$ at $70^{\circ} \mathrm{C}$.

\subsection{DNA sequencing}

Out of the 55 sampled animals, twelve sheep with different phenotypes (eight normal and four hypotrichotic sheep) were randomly chosen for sequencing. The PCR products were loaded on an agarose gel (1\%) and recovered from the gel using the "DNA and gel band purification kit" (Amersham Pharmacia Biotech). The sequencing reactions were prepared in $20 \mu \mathrm{L}$ containing $10 \mathrm{ng}$ every $100 \mathrm{bp}$ of PCR product, $3.2 \mathrm{pmol}$ of primer (Tab. I) and $8 \mu \mathrm{L}$ terminator ready reaction mix (DNA sequencing kit, Applied Biosystems). After an initial denaturation of $2 \mathrm{~min}$ at $94^{\circ} \mathrm{C}, 30$ cycles of $30 \mathrm{~s}$ at $96^{\circ} \mathrm{C}, 15 \mathrm{~s}$ at $50^{\circ} \mathrm{C}$, and $5 \mathrm{~min}$ at $60^{\circ} \mathrm{C}$ were performed. The extension products were purified from unincorporated dye-labeled terminators by "micro bio-spin P-30 tris chromatography columns" (Bio-Rad) and were analyzed using the automatic sequencer ABI PRISM 310 Genetic Analyzer (Perkin-Elmer Corporation, USA).

\subsection{SSCP analysis}

A PCR-SSCP (single strand conformation polymorphism) test was developed in order to differentiate the animals on the basis of the point mutation observed at the position 1312 of exon 3. The following primers were used:

\section{forward: 5'-GCCCAGCTGCCAGCCCGCAA-3' reverse: 5'-ACTGAGTCCAGCACTTCCTGCC-3'.}

Four microliters of the PCR product were mixed with $6 \mu \mathrm{L}$ formamide dye ( $95 \% \mathrm{w} / \mathrm{v}$ formamide, $0.025 \% \mathrm{w} / \mathrm{v}$ bromphenolblue, $0.025 \% \mathrm{w} / \mathrm{v}$ xylenecyanol FF, $20 \mathrm{mM}$ EDTA). They were denatured for $7 \mathrm{~min}$ at $95^{\circ} \mathrm{C}$ and were immediately chilled on ice. The samples were run for $15 \mathrm{~h}$ at $200 \mathrm{~V}$ and $12^{\circ} \mathrm{C}$ on a $15 \%$ acrylamide: bisacrylamide gel (37:1) with $0.5 \%$ glycerol. The bands were visualized by silver staining using the protocol of Bassam et al. [3]. 
The method was applied for typing of 43 other Valle del Belice individuals sampled corresponding to the different phenotypes (normal: $\mathrm{n}=31$; hypotrichotic $\mathrm{n}=12$ ), besides the sequenced animals, which were used as reference samples.

\subsection{Phylogenetic analysis}

The obtained DNA sequences were analyzed with the Blast program (www.ncbi.nlm.nih.gov). The comparison between the ovine sequences and the available hairless sequences of men, mice, and rats allowed the alignment of the ovine $h r$ coding sequences, and the evaluation of distances (percent divergence) between all pairs of sequences, using the ClustalW program.

\section{RESULTS}

\subsection{Amplification and sequencing}

A large portion of the entire $h r$ gene was successfully amplified using the long PCR. Sequencing of the region between exons 2 and 9, representing about $62 \%$ of the coding sequence, was obtained (accession number AY130969) for twelve unrelated sheep with different phenotypes. The analysis of the sequences revealed the occurrence of different mutations in exon 3 related to the hypotrichotic genotype: an A/T transversion at position 739, a G/A transition at position 823 , and a $\mathrm{C} / \mathrm{T}$ transition at position 1312. From these nucleotide exchanges, three substitutions of the processed mature protein were deduced at the amino acid positions 247 (Thr/Ser), 275 (Ala/Thr), and 438 (Gln/Stop).

Table II shows the phenotype of the sequenced animals, and the observed genotype at position 739, 823, and 1312 of the hairless gene exon 3. The correlation between the presence of the genotype homozygous for the mutated nucleotide and the presence of the hypotrichotic phenotype was $84 \%$ for the first two positions, and raised to $100 \%$ for the third point mutation.

\subsection{Genotyping}

The PCR-SSCP developed test allowed the detection of the genotype at the third mutation point (1312 position). The three genotypes CC, CT, and TT were clearly identifiable from the different electrophoretic patterns (Fig. 1). The $\mathrm{T}$ nucleotide was associated with a faster band, while a slower band was related to the $\mathrm{C}$ nucleotide. Both bands were clearly detectable in heterozygotes (samples 1 and 11).

The T nucleotide, resulting in the TAG stop codon in homozygotes, was always associated with the hypotrichotic phenotype. In fact, the correlation 
Table II. Phenotype of the sequenced animals and observed genotypes at positions 739,823 , and 1312 of the hairless gene exon 3.

\begin{tabular}{lcccc}
\hline Animal & Phenotype & 739 & 823 & 1312 \\
\hline 1 & Normal & AA & GG & CC \\
2 & Normal & TT & AA & CC \\
3 & Normal & AA & GG & CC \\
4 & Normal & AT & AG & CC \\
5 & Normal & AT & GG & CT \\
6 & Normal & AT & GG & CC \\
7 & Normal & AT & AG & CC \\
8 & Normal & AT & AG & CT \\
9 & Hypotrichotic & TT & AA & TT \\
10 & Hypotrichotic & TT & AA & TT \\
11 & Hypotrichotic & TT & AA & TT \\
12 & Hypotrichotic & TT & AA & TT \\
\hline
\end{tabular}

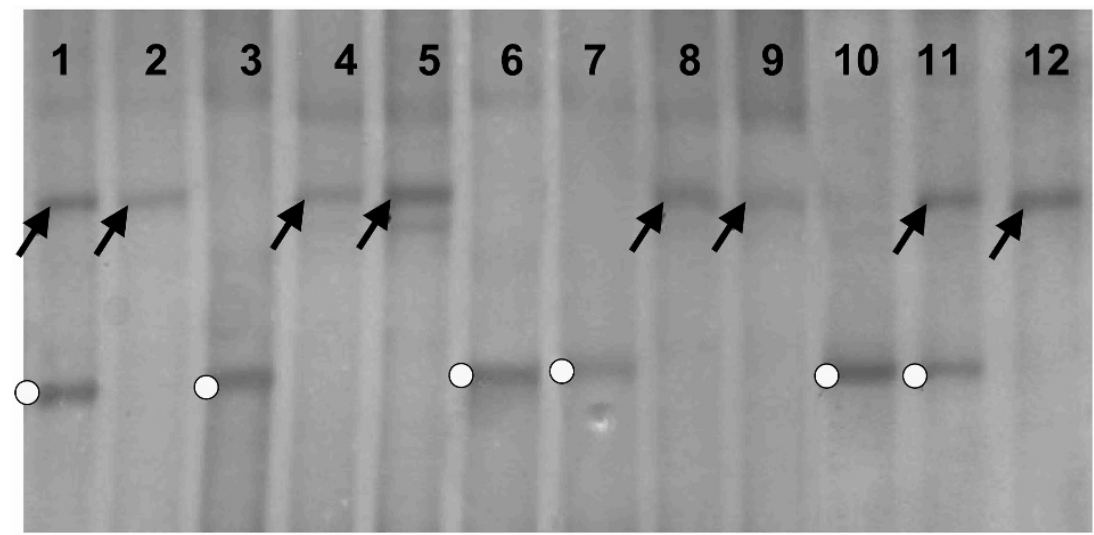

Figure 1. PCR-SSCP analysis for the detection of the mutation at nucleotide position 1312. The arrow and the dot indicate the bands related to the $\mathrm{C}$ (arrow) and $\mathrm{T}(\mathrm{dot})$ nucleotides. The genotypes of the sampled animals are as follows: CC (animals 2, 4, $5,8,9,12$ ); CT (animals 1, 11); TT (animals 3, 6, 7, 10).

between the occurrence of the TT genotype and the presence of the hypotrichotic phenotype was $100 \%$ on the whole sample, consisting of the 12 sequenced animals and of the other 43 animals genotyped by PCR-SSCP. All 16 hypotrichotic individuals presented an electrophoretic pattern associated with the TT genotype (Fig. 1). Among the 39 normal animals, 30 were found to be homozygous for the $\mathrm{C}$ nucleotide, while 9 were heterozygous (CT). 
Table III. Comparison among human, mouse and rat sequences and the two most frequent ovine haplotypes (sheep 1 and sheep 9 of Tab. III) for the observed exon 3 point mutations.

\begin{tabular}{lccccc}
\hline Position in sheep & Human & Mouse & Rat & Sheep 1 & Sheep 9 \\
\hline Codon 739-741 & TCA & TCA & TCG & ACT & TCT \\
Amino Acid 247 & Ser & Ser & Ser & Thr & Ser \\
\hline Codon 823-825 & CCC & CCT & CCT & GCC & ACC \\
Amino Acid 275 & Pro & Pro & Pro & Ala & Thr \\
\hline Codon 1312-1314 & CAG & CAG & CAG & CAG & TAG \\
Amino Acid 438 & Gln & Gln & Gln & Gln & Stop \\
\hline
\end{tabular}

\subsection{Phylogenetic analysis}

The available hairless sequences of men, mice, and rats were continuously aligned with the ovine $h r$ coding sequences, from exon 2 to exon 9, corresponding to $62 \%$ of the $h r$ coding sequence.

The comparison among these sequences and the two most frequent haplotypes for the three observed point mutations in the sequenced sheep, carried at the homozygous condition by animals 1 and 9 of Table II, is shown in Table III. The data suggest that the original ancestral haplotype of exon 3 carried the nucleotide combination T (739), C (823), and C (1312). Regarding the first point mutation (the 739 nucleotide position), the TCT codon may be the original sequence in ovines due to the presence of the same $\mathrm{T}$ nucleotide in the first position of the corresponding human, mouse and rat codon, and to the occurrence of the same amino acid (serine) in the processed mature protein. The evolutionary pathway is more uncertain for the second point mutation (the 823 nucleotide position), where both the exchanged nucleotides in the ovine codon (A and $\mathrm{G}$ ) do not occur in the corresponding codon position of the three other species. Moreover, the amino acids (threonine or alanine) deduced for the ovine processed mature protein are both different from proline, always occurring in humans, mice, and rats. The third nucleotide position (1312) was conserved in all the sequences except for the haplotype of sheep 9, where it always occurred in the hypotrichotic phenotype in the homozygous state. In fact, the CAG triplet, coding for Gln in humans, mice, rats and sheep 1, was mutated in the stop codon TAG in sheep 9 as well as in the other hypotrichotic sheep.

The alignment of the obtained ovine sequences showed the highest homology (72.9\%) with the human $h r$ gene (Tab. IV). The high values of the homology scores, ranging from $63.1 \%$ to $90.5 \%$, indicate that the hairless gene is strongly conserved. 
Table IV. Percentage of homology between the aligned sequences.

\begin{tabular}{lcccc}
\hline Species & Homo & Ovis & Mus & Rattus \\
\hline Homo & 100 & 72.9 & 71.9 & 69.8 \\
Ovis & & 100 & 66.9 & 63.1 \\
Mus & & & 100 & 90.5 \\
Rattus & & & & 100 \\
\hline
\end{tabular}

The phylogenetic analysis agrees with the well known evolutionary pathway of mammals [8], indicating that the divergences of the $h r$ gene in ruminants, rodents, and primates stem from a common ancestor.

\section{DISCUSSION}

These results clearly demonstrate that the $h r$ gene is involved in the genetic control of the congenital hypotrichosis in the ovine Valle del Belice breed. Among the observed nucleotide exchanges, the stop codon at position 1312 was found to be the causative mutation of the metabolic disorder. Two other nucleotide exchanges were found to be closely linked to the mutation responsible for the lack of hair, resulting in at least five different haplotypes. The most common haplotypes were A (739), G (823), C (1312), usually occurring in normal animals, and T (739), A (823), T (1312) always observed in the homozygous state in the hypotrichotic animals. Two other haplotypes deduced from the informative genotypes (animal 2 and 4 of Tab. II) were T-A-C and T-G-C. Animal 5 reveals the occurrence of another haplotype, which could be T-G-T (A-G-C on the other DNA strand), or A-G-T (T-G-C on the other strand).

The high number of different haplotypes suggests a very high frequency of recombination and gene conversion in exon 3. Moreover, the most spread haplotype in the sequenced sheep is different at the amino acid position 247 and 275 from the other mammalian sequences considered (Tab. III). The high variability of exon 3 is in contrast with the low mutation rate and the reduced phylogenetic divergence of the $h r$ gene in the analyzed species (Tab. IV). These data suggest the existence of a specific mechanism for the local production of genetic variation in exon 3, and the $h r$ exon 3 haplotype structure, both in Valle del Belice breed and in other sheep populations, is under further investigation.

The mutations discovered in exon 3 may be responsible not only for the hypotrichotic phenotype but also for other important functional variations in different metabolic traits. This hypothesis agrees with recent experimental evidence indicating that the hairless gene product plays a wider role in development than previously suspected [5]. Several pleiotropic effects were detected 
in different mouse tissues such as cartilage, developing tooth, the inner ear, retina, colon, skin, and brain.

Potter et al. [13] demonstrated that the $\mathrm{Hr}$ protein encoded by the hairless gene functions as a transcriptional corepressor for thyroid hormone receptor (TR). $\mathrm{Hr}$ binds to TR and interacts with histone deacetylases. On the basis of its thyroid hormone-inducible and tissue specific expression, $\mathrm{Hr}$ likely defines a new class of nuclear receptor corepressors that serve an important role in the corepressor machinery. Therefore the investigation of the hairless gene in domestic animals could reveal interesting influences on productive traits in addition to the well-known effect on hair growth.

\section{ACKNOWLEDGEMENTS}

This work was funded through grants from ex-MURST $60 \%$ and CNRAgenzia2000.

\section{REFERENCES}

[1] Ahmad W., Haque M.F., Brancolini V., Tsou H.C., Haque S., Lam H., Aita V.M., Owen J., de Blaquiere M., Frank J., Cserhalmi-Friedman P.B., Leask A., McGrath J.A., Peacocke M., Ahmad M., Ott J., Christiano A.M., Alopecia universalis associated with a mutation in the human hairless gene, Science 279 (1998) 720-724.

[2] Ahmad W., Zlotogorski A., Panteleyev A., HaMut L., Ahmad M., Faiyaz ul Haque M., Abdallah H., Dragan L., Christiano A.M., Genomic organization of the hairless gene (HR) and identification of a mutation underlying congenital atrichia in an Arab Palestinian family, Genomics 56 (1999) 141-148.

[3] Bassam B.J., Caetano-Anolles G., Gresshoff P.M., Fast and sensitive silver staining of DNA in polyacrylamide gels, Anal. Biochem. 196 (1991) 80-83.

[4] Cachon-Gonzalez M., Fenner S., Coffin J., Moran C., Best S., Stoye J., Structure and expression of the hairless gene of mice, Proc. Natl. Acad. Sci. USA 91 (1994) 7717-7721.

[5] Cachon-Gonzalez M., San-Josè I., Cano A., Vega J.A., García N., Freeman T., Schimmang T., Stoye J., The hairless gene of the mouse: Relationship of phenotypic effects with expression profile and genotype, Dev. Dyn. 216 (1999) $113-126$.

[6] Cichon S., Anker M., Vogt I.R., Rohleder H., Putzstuck M., Hillmer A., Farooq S.A., Al-Dhafri K.S., Ahmad M., Haque S., Rietschel M., Propping P., Kruse R., Nothen M.M., Cloning, genomic organization, alternative transcripts and mutational analysis of the gene responsible for autosomal recessive universal congenital alopecia, Hum. Mol. Genet. 7 (1998) 1671-1679.

[7] Dolling C., Brooker M., A viable hypotrichosis in Poll Dorset sheep, J. Hered. 57 (1966) 87-90. 
[8] Easteal S., Molecular evidence for the early divergence of placental mammals, Bioessays 21 (1999) 1052-1058.

[9] Finocchiaro R., Portolano B., Castiglioni B., Sironi G., Madonia G., Domeneghini C., Pagnacco G., Descrizione preliminare di pecore della Valle del Belice affette da alopecia ereditaria, in: Proceedings S. I. P. A. O. C., 18-21 October 2000, Vol. 1, Vietri sul Mare (SA), pp. 295-298.

[10] Mackie J., McIntyre B., Congenital hypotrichosis in Poll Dorset sheep, Aust. Vet. J. 69 (1992) 146-147.

[11] Nel J., Congenital hypotrichosis in Karakul sheep, S. Afr. Agric. Sci. 7 (1964) 875-877.

[12] Panteleyev A., Botchkareva N., Sundberg J., Christiano A., Paus R., The role of the hairless $(h r)$ gene in the regulation of hair follicle catagen transformation, Amer. J. Pathol. 155 (1999) 159-171.

[13] Potter G.B., Beaudoin G.M. 3rd, DeRenzo C.L., Zarach J.M., Chen S.H., Thompson C.C., The hairless gene mutated in congenital hair loss disorders encodes a novel nuclear receptor corepressor, Genes Develop. 15 (2001) 2687-2701.

To access this journal online: www.edpsciences.org 